\title{
Effective Viscosity of Confined Hydrocarbons
}

\author{
I.M. Sivebaek ${ }^{1,2,3}$, V.N. Samoilov ${ }^{1,4}$ and B.N.J. Persson ${ }^{1}$ \\ ${ }^{1}$ IFF, FZ-Jülich, 52425 Jülich, Germany \\ ${ }^{2}$ Novo Nordisk A/S, Research and Development, DK-3400 Hillerod, Denmark \\ ${ }^{3}$ Mech. Eng. Dept., Technical University of Denmark, DK-2800 Lyngby, Denmark and \\ ${ }^{4}$ Physics Faculty, Moscow State University, 117234 Moscow, Russia
}

\begin{abstract}
We present Molecular Dynamics (MD) friction calculations for confined hydrocarbon films with molecular lengths from 20 to 1400 carbon atoms. We find that the logarithm of the effective viscosity $\eta_{\text {eff }}$ for nanometer-thin films depends linearly on the logarithm of the shear rate: $\log \eta_{\text {eff }}=C-n \log \dot{\gamma}$, where $n$ varies from 1 (solid-like friction) at very low temperatures to 0 (Newtonian liquid) at very high temperatures, following an inverse sigmoidal curve. Only the shortest chain molecules melt, whereas the longer ones only show a softening in the studied temperature interval $0<T<900 \mathrm{~K}$. The results are important for the frictional properties of very thin (nanometer) films and to estimate their thermal durability.
\end{abstract}

The frictional and rheological properties of thin confined fluid films are of great importance in a number of engineering and scientific applications, e.g., in the context of lubrication. Thus, recent studies [1] have shown that for sliding contacts lubricated by organic or silicon oil bulk shear thinning can have a great influence on the friction at intermediate sliding velocities (mixed lubrication). When wetting fluids are confined between solid walls at nanometer separation they often acquire solidlike properties and an increasing squeezing force is necessary in order to reduce the film thickness. If the solid walls are smooth the fluid molecules arrange in layers parallel to the solid walls and the squeeze-out occurs in a quantized way by removing one monolayer after another with increasing pressure $2-4]$. Sometimes the last one or two monolayers are so strongly bound that they cannot be removed by squeezing alone. Fluid films confined at the nanometer length scale exhibit viscosity enhancement and nonlinear flow properties characteristic of sheared supercooled liquids approaching its glass transition [5, 6] .

Experiments for a large variety of fluids (including hydrocarbon fluids and silicon oil) [4, 7] have shown that the logarithm of the effective viscosity $\eta_{\text {eff }}$ for nanometer-thin films (typically 3 or 4 monolayers and contact pressures of the order of a few $\mathrm{MPa}$ ) depends linearly on the logarithm (here and elsewhere with 10 as the basis) of the shear rate: $\log \eta_{\text {eff }}=C-n \log \dot{\gamma}$. If $\eta_{\text {eff }}$ and $\dot{\gamma}$ are measured in SI units, for a large variety of fluids (at room temperature) $C \approx 5$ and $n \approx 0.9$. This linear relation has also been established in other experiments [8, 9] and in computer simulations [10, 11].

We have performed a very extensive set of Molecular Dynamics (MD) simulations to probe the frictional properties of thin layers of confined hydrocarbon molecules (with molecular lengths from 20 to 1400 carbon atoms). Some of these results may also be relevant for polymeron-polymer systems as recent studies [12] have shown that in this case the shear deformations are localized to a band of material about $2.5 \mathrm{~nm}$ thick. Our results for $n$ and $C$ agree with the experimental observation at room temperature, but show that when the temperature increases $n$ varies from 1 (solid-like friction) at very low temperatures to 0 (Newtonian liquid) at very high temperatures.

Our model is similar to those described in Refs. [13, 14]. We consider a block and a substrate with atomically flat surfaces separated by a polymer slab consisting of hydrocarbons with molecular lengths 20, 100 and 1400 carbon atoms. The solid walls are treated as single layers of "atoms" bound to rigid flat surfaces by springs corresponding to the long-range elastic properties of $50 \AA$ thick solid slabs similar to our earlier papers (see, e.g. Refs. [15 17]). The simulation box in $x-y$ dimension is equal to $124.8 \AA \times 124.8 \AA$. In the following, periodic boundary conditions are assumed in the $x y$ plane.

Initially about half of the molecules are adsorbed on the block surface and half on the substrate surface. Two solids with adsorbed polymer slabs were put into contact and when the temperature was equal to the thermostat temperature everywhere we started to move the upper block surface. The temperature was varied from $0 \mathrm{~K}$ to $900 \mathrm{~K}$ to study the effect of temperature (and also melting) on the shear stress. In our simulations, the polymer films are very thin $(\sim 3 \mathrm{~nm})$, and the solid walls are connected to a thermostat at a short distance from the polymer slab. Under these circumstances we find that frictional heating effects are not important, and the effective temperature in the polymer film is always close to the thermostat temperature.

Linear alkanes $\mathrm{C}_{n} \mathrm{H}_{2 n+2}$ (with $n=20,100$ and 1400) were used as "lubricant" in the present calculations. The $\mathrm{CH}_{2} / \mathrm{CH}_{3}$ beads are treated in the united atom representation [18, 19]. The Lennard-Jones potential was used to model the interaction between beads of different chains

$$
U(r)=4 \epsilon_{0}\left[\left(\frac{r_{0}}{r}\right)^{12}-\left(\frac{r_{0}}{r}\right)^{6}\right]
$$

and the same potential with modified parameters $\left(\epsilon_{1}, r_{1}\right)$ was used for the interaction of each bead with the substrate and block atoms. 
The parameters were $\epsilon_{0}=5.12 \mathrm{meV}$ for both the interior and the end beads, and $r_{0}=3.905 \AA$. For the interactions within the $\mathrm{C}_{n} \mathrm{H}_{2 n+2}$ molecules we used the standard optimized potentials for liquid simulations model [18, 19], including flexible bonds, bond bending and torsion interaction, which results in bulk properties in good agreement with experimental data far below the boiling point [20]. Atomic mass 14 (for interior $\mathrm{CH}_{2}$ beads) and 15 (for the $\mathrm{CH}_{3}$ end groups) were used. Within a $\mathrm{C}_{n} \mathrm{H}_{2 n+2}$ chain we assume nearest neighbor $\mathrm{C}$ atoms are connected via springs with the spring constant $k$, which was chosen equal to $10 \mathrm{~N} / \mathrm{m}$. Note that this value is one order of magnitude smaller then the optimized $450 \mathrm{~N} / \mathrm{m}$ [18], and was chosen such to facilitate a reasonable time step of $1 \mathrm{fs}$. We used an angle bending interaction of the form $E(\cos \theta) / k_{B}=(1 / 2) k_{\text {bend }}\left(\cos \theta-\cos \theta_{0}\right)^{2}$ with $k_{\text {bend }}=62543 \mathrm{~K}$ and $\theta_{0}=2.0001 \mathrm{rad}$. For the dihedral interaction we used the functional form in term of a cosine Fourier series $E(\phi) / k_{B}=\sum_{i=0}^{3} c_{i} \cos ^{i}(\phi)$ with parameters $c_{0}=1009.99 \mathrm{~K}, c_{1}=2018.95 \mathrm{~K}, c_{2}=136.37 \mathrm{~K}$, $c_{3}=-3165.30 \mathrm{~K}$. Internal beads of separation greater than 3 units are treated similarly as beads from different chains. The number of molecules was equal to 1000, 200 and 14 for the $\mathrm{C}_{20} \mathrm{H}_{42}, \mathrm{C}_{100} \mathrm{H}_{202}$ and $\mathrm{C}_{1400} \mathrm{H}_{2802}$ systems respectively. The hydrocarbon films at room temperature consisted of 6 to 8 monolayers of molecules between the solid surfaces. The (nominal) squeezing pressure $p_{0}$ was usually $10 \mathrm{MPa}$.

We have chosen the polymer-wall atom bond to be so strong that no slip occurs at these interfaces. This is the case with $r_{1}=2.92 \AA, \epsilon_{1}=160 \mathrm{meV}$. The lattice spacings of the block and of the substrate are $a=b=$ $2.6 \AA$.

If $v$ is the sliding velocity and $d$ the film thickness, we define the shear rate $\dot{\gamma}=v / d$ and the effective viscosity $\eta_{\text {eff }}=\sigma_{\mathrm{f}} / \dot{\gamma}$, where $\sigma_{\mathrm{f}}$ is the frictional shear stress. Fig. 1 shows the logarithm of the effective viscosity as a function of the temperature 21] for the (a) $\mathrm{C}_{20} \mathrm{H}_{42}$, (b) $\mathrm{C}_{100} \mathrm{H}_{202}$ and (c) $\mathrm{C}_{1400} \mathrm{H}_{2802}$ system, at four sliding velocities (from top to bottom) $0.3,3,10$ and $100 \mathrm{~m} / \mathrm{s}$. Increasing the velocity results in a reduced effective viscosity, i.e., the thin films exhibit shear thinning.

The velocity dependence of the MD data in Fig. 1 can be very well fitted by the formula $\eta_{\mathrm{eff}}=B \dot{\gamma}^{-n}$ or

$$
\log \eta_{\text {eff }}=C-n \log \dot{\gamma},
$$

where $C=\log B$. This is illustrated in Fig. 2 for two cases. The rest of the cases also show that a linear fit is appropriate with the exceptions of the results at very high temperatures. The $\mathrm{C}_{100} \mathrm{H}_{202}$ system shows the highest effective viscosity due to entanglement. The $\mathrm{C}_{20} \mathrm{H}_{42}$ is nearly liquid above $300 \mathrm{~K}$ and shows less entanglement whereas the sliding in $\mathrm{C}_{1400} \mathrm{H}_{2802}$ takes place at nearly one interface (see figure 6). We find that the parameters $C$ and $n$ depend on the temperature. The data points in Fig. 3 show the temperature dependence of the index $n$,

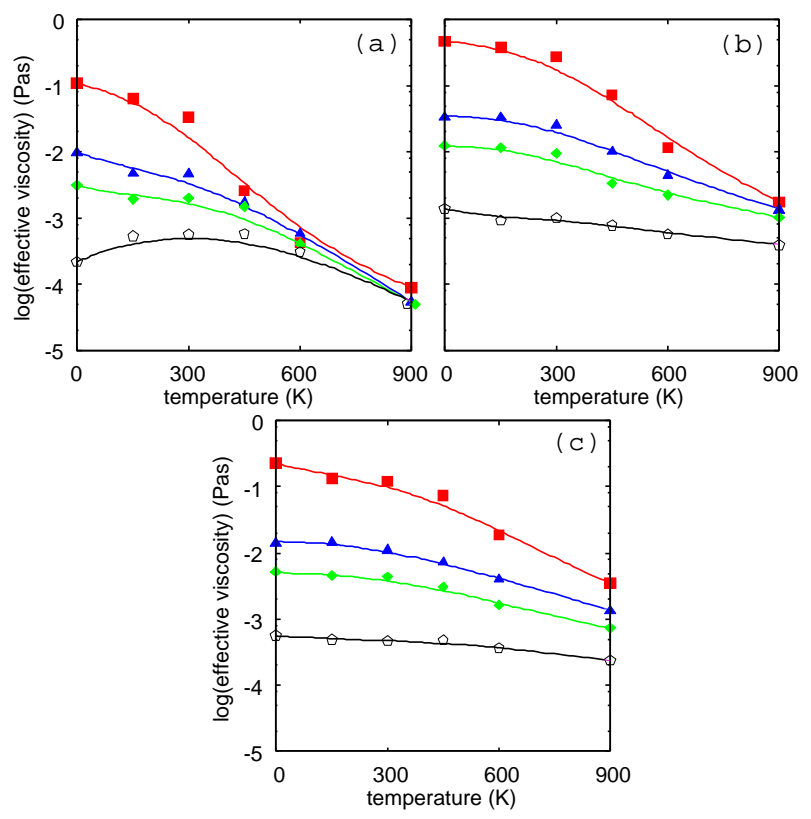

FIG. 1: (Color online) The logarithm of the effective viscosity as a function of the temperature for (a) $\mathrm{C}_{20} \mathrm{H}_{42}$, (b) $\mathrm{C}_{100} \mathrm{H}_{202}$ and (c) $\mathrm{C}_{1400} \mathrm{H}_{2802}$ system at four sliding velocities: $0.3 \mathrm{~m} / \mathrm{s}$ $(\boldsymbol{\square}), 3 \mathrm{~m} / \mathrm{s}(\boldsymbol{\Delta}), 10 \mathrm{~m} / \mathrm{s}(\diamond)$ and $100 \mathrm{~m} / \mathrm{s}(\bullet)$.

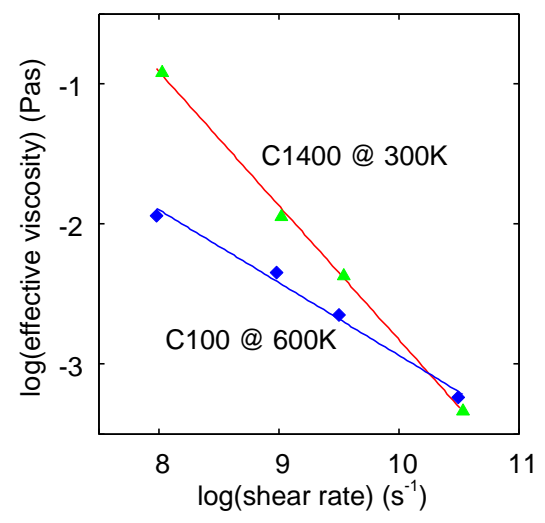

FIG. 2: (Color online) The data points for the logarithm of the effective viscosity as a function of the logarithm of the shear rate at four sliding velocities: $0.3,3,10$ and $100 \mathrm{~m} / \mathrm{s}$. The lines represent the linear fits to these data points. The systems are $\mathrm{C}_{100} \mathrm{H}_{202}$ at $600 \mathrm{~K}$ and $\mathrm{C}_{1400} \mathrm{H}_{2802}$ at $300 \mathrm{~K}$. The values of the slope and the intercept of the $\mathrm{C}_{100} \mathrm{H}_{202}$ are $-0.520 \pm 0.038$ and $2.260 \pm 0.351$ and for the $\mathrm{C}_{1400} \mathrm{H}_{2802}$ system $-0.958 \pm 0.024$ and $6.753 \pm 0.228$.

while the solid lines are fits to the data points using the inverse sigmoidal curve:

$$
n=\frac{1}{1+\left(T / T_{c}\right)^{\alpha}} .
$$

The parameters $\alpha$ and $T_{c}$ are given in table I. At low temperatures $n=1$ as expected for dry friction. That is, the frictional shear stress $\sigma_{\mathrm{f}}=\eta_{\mathrm{eff}} \dot{\gamma}=10^{C} \dot{\gamma}^{1-n}$ is independent of the shear rate when $n=1$ as expected for 


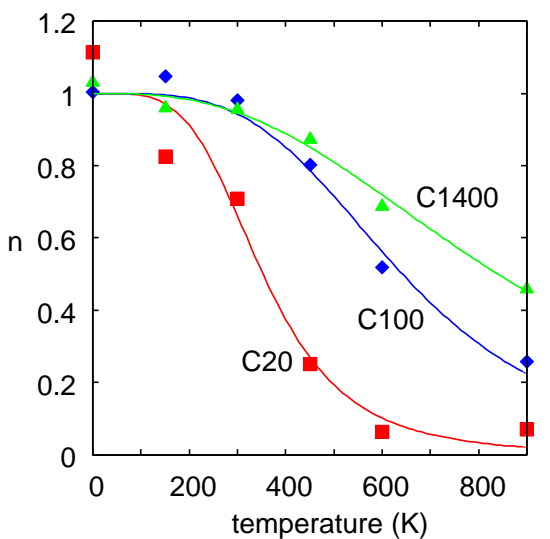

FIG. 3: (Color online) Values of the parameter $n$ in equation (2) as a function of the temperature for the systems $\mathrm{C}_{20} \mathrm{H}_{42}$, $\mathrm{C}_{100} \mathrm{H}_{202}$ and $\mathrm{C}_{1400} \mathrm{H}_{2802}$. The solid lines are fits to the numerical data using equation (3) with the parameters $\alpha$ and $T_{c}$ given in table \.

\begin{tabular}{|c|c|c|c|}
\hline & $\mathrm{C}_{20} \mathrm{H}_{42}$ & $\mathrm{C}_{100} \mathrm{H}_{202}$ & $\mathrm{C}_{1400} \mathrm{H}_{2802}$ \\
\hline$T_{c}(\mathrm{~K})$ & $353 \pm 28$ & $642 \pm 22$ & $840 \pm 30$ \\
\hline$\alpha(-)$ & $4.09 \pm 1.24$ & $3.68 \pm 0.46$ & $2.79 \pm 0.30$ \\
\hline
\end{tabular}

TABLE I: Table showing the $T_{c}$ and $\alpha$ in equation (3) for each system. The standard deviation of the fitting parameters is also indicated.

dry friction at low temperatures (no thermally activated creep). At high temperature $n$ approaches 0 as expected for a Newtonian fluid where the frictional shear stress is proportional to the shear rate.

The parameter $C$ depends on the units used for $\eta_{\text {eff }}$ and $\dot{\gamma}$ and here we assume SI units. Remarkably we find a linear relation between $C$ and $n$ for all the systems and temperatures we have studied, see Fig. 4. The extrapolation of $C$ to $n=0$ gives $C \approx-3.8 \pm 0.2$. This gives the effective viscosity $\eta_{\text {eff }}=10^{C}=(1.6-2.5) \times 10^{-4}$ Pas. Experiments have shown 22 that for a wide range of fluids the fluid viscosity at the boiling point is $\approx 2.2 \times 10^{-4} \mathrm{Pas}$, i.e., nearly the same as we deduce for confined fluids when extrapolating our data to $n=0$ (Newtonian fluid). This result is extremely interesting but perhaps not entirely unexpected, as $n=0$ corresponds to high temperatures where the separation between the solid walls (see Fig. 5 in which the sliding velocity is $10 \mathrm{~m} / \mathrm{s}$ ) is much larger than for lower temperatures and where the mobility of the fluid molecules may be similar to that in the bulk fluid close to the boiling point. The $\mathrm{C}_{100} \mathrm{H}_{202}$ film has a smaller film thickness than the other systems at low temperatures due to the fact that it only has 6 monolayers at these conditions. The other two systems have 7 monolayers at the same conditions and have thus thicker films. This matter has been discussed in reference 14.

Fig. 3 shows that the longer the hydrocarbon chain length the higher the temperature necessary for the film

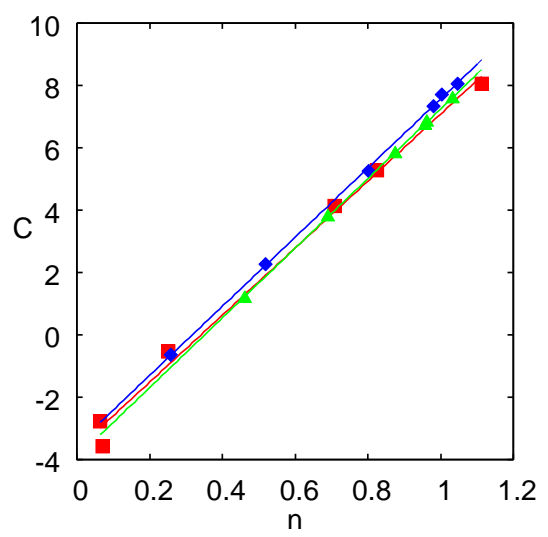

FIG. 4: (Color online) The $C$ parameter in equation (2) also follows a sigmoidal curve when the temperature is varied. This figure shows the dependence of $C$ on $n$. All three systems $\mathrm{C}_{20} \mathrm{H}_{42}, \mathrm{C}_{100} \mathrm{H}_{202}$ and $\mathrm{C}_{1400} \mathrm{H}_{2802}$ are represented.

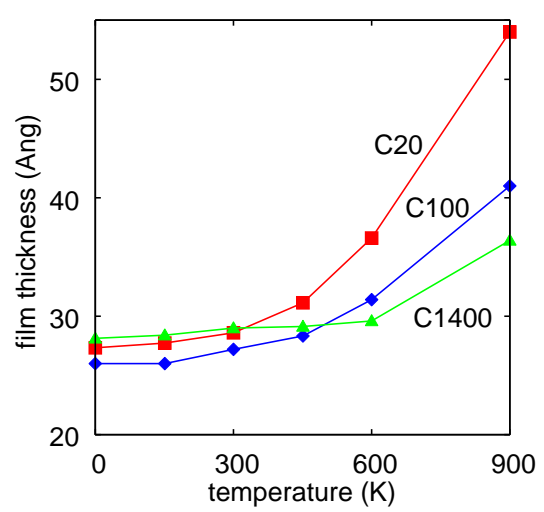

FIG. 5: (Color online) The polymer film thickness as a function of the temperature. The sliding speed is $10 \mathrm{~m} / \mathrm{s}$. All three systems $\mathrm{C}_{20} \mathrm{H}_{42}, \mathrm{C}_{100} \mathrm{H}_{202}$ and $\mathrm{C}_{1400} \mathrm{H}_{2802}$ are represented.

to behave as a Newtonian fluid. This is also illustrated in Fig. 6] which shows the relative number of atoms traveling at velocities below $v$, as a function of $v$, for all three polymer films at $600 \mathrm{~K}$. It can be seen that the velocity gradient is going from nearly Newtonian in the case of $\mathrm{C}_{20} \mathrm{H}_{42}$ to solid-like in the $\mathrm{C}_{1400} \mathrm{H}_{2802}$ case.

Note that for the hydrocarbons $\mathrm{C}_{100} \mathrm{H}_{202}$ and $\mathrm{C}_{1400} \mathrm{H}_{2802}$ at room temperature $n \approx 0.9$, as also found experimentally for many confined fluids. When $n \approx 0.9$ from Fig. 4 we get $C \approx 6$ which is a little larger than found experimentally (at room temperature) $C_{\exp } \approx 5$. This may reflect somewhat different confinement condition, e.g., differences in contact pressures $(10 \mathrm{MPa}$ in our study as compared to a few MPa in most of the experimental studies). However, in both cases the contact pressure is so small that one does not expect any significant dependency of the shear stress on the contact pressure 13], except if there is a pressure induced change in the number of confined layers.

It is well known that the viscosity of fluids at high pres- 


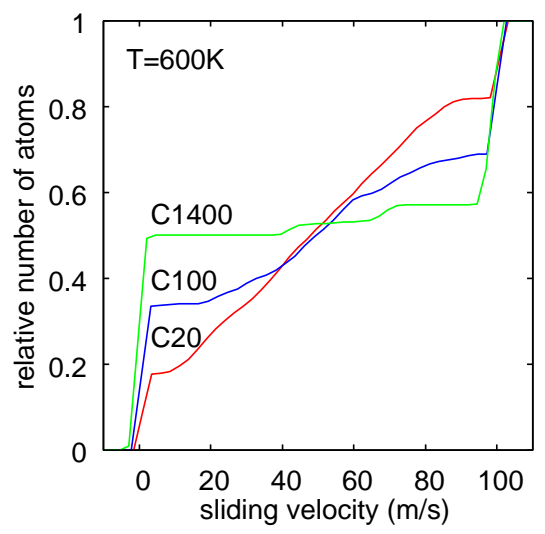

FIG. 6: (Color online) The relative number of atoms moving at the velocity below $v$, as a function of $v$, at $600 \mathrm{~K}$. It can be seen that the velocity gradient is going from nearly Newtonian in the case of $\mathrm{C}_{20} \mathrm{H}_{42}$ to solid-like in the $\mathrm{C}_{1400} \mathrm{H}_{2802}$ case.

sures may be many orders of magnitude larger than at low pressures. Using the theory of activated processes, and assuming that a local molecular rearrangement in a fluid results in a local volume expansion, one expects an exponential dependence of viscosity $\eta$ on the hydrostatic pressure $p, \eta=\eta_{0} \exp \left(p / p_{0}\right)$, where typically (for hydrocarbons or polymer fluids) $p_{0} \approx 10^{8} \mathrm{~Pa}$ (see, e.g., Refs. [23, 24]). Here we are interested in (wetting) fluids confined between the surfaces of elastically soft solids, e.g., rubber. In this case the pressure at the interface is usually at most of the order of the Young's modulus, which (for rubber) is less than $10^{7} \mathrm{~Pa}$. Thus, in most cases involving elastically soft materials, the viscosity can be considered as independent of the local pressure.

One of us have recently studied rubber friction on rough surfaces [25]. For unfilled styrene butadiene rubber we found the transfer of a thin smear film to the substrate. In this case the shear during sliding may be localized to a thin (a few nanometer) interfacial layer which may exhibit frictional properties very similar to what we have observed in our simulations. Indeed the experimental data indicated a frictional shear stress of the form predicted above with $n \approx 0.91$ and $10^{C} \approx 1.3 \times 10^{5}$ (in SI units), in close agreement with the result of our simulations.

To summarize, we have presented results of molecular dynamics calculations of friction performed for a block sliding on a substrate separated by $\approx 3 \mathrm{~nm}$ thick polymer films where the alkanes had 20, 100 and 1400 carbon atoms. In all cases we found that the logarithm of the effective viscosity is proportional to the logarithm of the shear rate, $\log \eta_{\mathrm{eff}} \approx C-n \log \dot{\gamma}$. The index $n$ varies from 1 (solid-like friction) at very low temperatures to 0 (Newtonian liquid) at very high temperatures, following an inverse sigmoidal curve. The $C$ parameter is proportional to $n$ and as $n \rightarrow 0,10^{C}$ extrapolates to the viscosity of the bulk fluid at the boiling point. At room temperature the parameters $n$ and $C$ have been found to be close to what has been observed experimentally for a large number of fluids.

Two of the authors (I.M.S. and V.N.S.) acknowledge support from IFF, FZ-Jülich, hospitality and help of the staff during their research visits. I.M.S. acknowledges the 2010 Jacob Wallenberg Prize in Materials Science from the Royal Swedish Academy of Engineering Sciences.

[1] B.N.J. Persson and M. Scaraggi, Eur. Phys. J. E 34, 113 (2011).

[2] E. Kumacheva and J. Klein, Journal of Chemical Physics 108, 16, 7010 (1998).

[3] B.N.J. Persson and F. Mugele, Journal of Physics: Condensed Matter 16, R295 (2004).

[4] L. Bureau, Phys. Rev. Lett. 104, 218302 (2010).

[5] M.D. Demetriou et al., Phys. Rev. Lett. 97, 065502 (2006).

[6] A. Furukawa, K. Kim, S. Saito and H. Tanaka, Phys. Rev. Lett. 102, 016001 (2009).

[7] S. Yamada, Tribology Letters 13, 167 (2002).

[8] S. Granick, Science 253, 1374 (1991).

[9] G. Luengo, F.-J. Schmitt, R. Hill and J. Israelachvili, Macromolecules 30, 8, 2482 (1997).

[10] P.A. Thompson, G.S. Grest and M.O. Robbins, Phys. Rev. Lett. 68, 23, 3448 (1992).

[11] M.O. Robbins and A.R.C. Baljon, in Microstructure and Microbiology of Polymer Surfaces, American Chemical Society,Washington, DC, ch. 6 (2000).

[12] Y.K. Yew, M. Minn, S.K. Sinha and V.B.C. Tan, Langmuir 27, 5891 (2011).

[13] I.M. Sivebaek, V.N. Samoilov and B.N.J. Persson, European Physical Journal E: Soft Matter 27, 37 (2008).

[14] I.M. Sivebaek, V.N. Samoilov and B.N.J. Persson, Langmuir 26, 8721 (2010).

[15] B.N.J. Persson, V.N. Samoilov, S. Zilberman and A. Nitzan, J. Chem. Phys. 117, 3897 (2002).

[16] I.M. Sivebaek, V.N. Samoilov and B.N.J. Persson, J. Chem. Phys. 119, 2314 (2003).

[17] V.N. Samoilov and B.N.J. Persson, J. Chem. Phys. 120, 1997 (2004).

[18] W.L. Jorgensen, J.D. Madura and C.J. Swenson, J. Am. Chem. Soc. 106, 6638 (1984).

[19] D.K. Dysthe, A.H. Fuchs and B. Rousseau, J. Chem. Phys. 112, 7581 (2000).

[20] M.G. Martin and J.I. Siepmann, J. Phys. Chem. B 102, 2569 (1998).

[21] The "temperature" was obtained from the total kinetic energy but even at the highest sliding velocity $100 \mathrm{~m} / \mathrm{s}$ the contribution from the center of mass motion to the "temperature" is not very large, corresponding to an increase in the "temperature" by only $\sim 15 \mathrm{~K}$, and much smaller at the lower sliding velocities (the contribution to the "temperature" from the translational motion scales as the square of the sliding velocity).

[22] A.W. Francis, Industrial and engineering chemistry 49, 1779 (1957).

[23] H. Eyring, J. Chem. Phys. 4, 283 (1936).

[24] B.N.J. Persson, Sliding Friction: Physical Principles and 
Applications, 2nd ed. (Springer, Heidelberg, 2000).

[25] B. Lorenz, B.N.J. Persson, S. Dieluweit and T. Tada,
Eur. Phys. J. E 34, 129 (2011). 